\title{
Dye-Sensitized Solar Cells Using Mesocarbon Microbead-Based Counter Electrodes
}

\author{
Chien-Te Hsieh, Bing-Hao Yang, and Wei-Yu Chen \\ Department of Chemical Engineering and Materials Science, Yuan Ze Fuel Cell Center, Yuan Ze University, Taoyuan 320, Taiwan \\ Correspondence should be addressed to Chien-Te Hsieh, cthsieh@saturn.yzu.edu.tw
}

Received 30 May 2011; Accepted 26 November 2011

Academic Editor: Peter Rupnowski

Copyright () 2012 Chien-Te Hsieh et al. This is an open access article distributed under the Creative Commons Attribution License, which permits unrestricted use, distribution, and reproduction in any medium, provided the original work is properly cited.

The dye-sensitized solar cells (DSCs) equipped with mesocarbon microbead (MCMB)-based counter electrodes were explored to examine their cell performance. Three types of nanosized additives including platinum, carbon nanotubes (CNTs), and carbon black $(\mathrm{CB})$ are well dispersed and coated over microscaled MCMB powders. In the design of the counter electrodes, the MCMB graphite offers an excellent medium that allows charge transfer from the ITO substrate to the dye molecule. The active materials such as Pt, CNT, and nanosize CB act as an active site provider for the redox reaction. Among these counter electrodes, the DSCs fabricated with $\mathrm{CB}$ electrode exhibit the highest power conversion efficiency. This improved efficiency can be attributed to the fact that the CB nanoparticles not only offer a large number of catalytic sites but also low charge transfer resistance, facilitating a rapid reaction kinetics. Such design of carbon counter electrode has been confirmed to be a promising candidate for replacing $\mathrm{Pt}$ electrodes.

\section{Introduction}

Dye-sensitized solar cell (DSC) is one of the representative organic solar cells, resembling a photosynthesis process in nature [1]. Up to now, DSC has drawn considerable attention due to its low-cost production, environmentally benign material, and facile fabrication, compared with Si-based solar cell [2-5]. A DSC is a typical electrochemical system, consisting of a wide bend gap semiconductor photoanode, a dye, an electrolyte, and a counter electrode [6]. It is generally recognized that nanocrystalline $\mathrm{TiO}_{2}$ is one of the suitable materials for the photoanode of DSCs. This is attributed to the fact that its edge of conduction band allows the electron injection from the excited state of dye. The function of counter electrode is to reduce $I_{3}{ }^{-}$to $I^{-}$in electrolyte by electron transferred from photoanode. Platinum coated on indium- or fluorine-coated tin oxide (ITO or FTO) conducting glass often serves as counter electrode in DSCs because the Pt counter electrode displays high electric conductivity and high electrochemical catalytic activity for triiodide reduction $[7,8]$. However, the Pt film can be decomposed to $\mathrm{PtI}_{4}$ compound by $\mathrm{I}_{3}{ }^{-} / I^{-}$electrolyte during the DSC operation, reducing the performance, long-term durability, and commercial success of DSCs. Additionally, to reduce the usage amount of noble Pt, seeking one cheap candidate to replace costly $\mathrm{Pt}$ is still a challenge.

More recently, early reports have pointed out that carbonaceous counter electrodes show a potential feasibility to replace Pt-based electrodes. Several carbon candidates including activated carbon paste [8], carbon nanotubes (CNTs)-based paste $[6,9,10]$, Pt-loaded carbon black [11, $12]$, and nanoscaled carbon powder $[13,14]$ have been proven to show the catalytic capability for reduction of $\mathrm{I}_{3}{ }^{-}$ions. However, a better understanding is required to optimize the cell performance using different carbon counter electrodes. In this paper, spherical mesocarbon microbead (MCMB) graphite has been selected as a matrix electrode material due to its good electric conductivity, stability, and easy coating. Actually, MCMB powders with microscaled size have been extensively used as anode materials in Liion battery. To improve the cell performance, three types of additives, Pt nanoparticles, CNTs, and carbon black (CB) 
nanoparticles, were well dispersed over MCMB graphite surface. The DSCs fabricated with different counter electrodes were examined using their incident photon to current efficiency (IPCE) spectra and photocurrent-voltage curves for comparison. This study aims to shed some lights on (i) how the carbon-based counter electrodes affect the performance of DSCs and (ii) the possibility of carbon counter electrodes to replace Pt electrodes in this photovoltaic device.

\section{Experimental}

Commercial MCMB (Osaka Gas Co., Japan) graphite powders, having an average size of $10 \mu \mathrm{m}$, were used as matrix. The MCMB sample was initially dispersed into ethylene glycol, forming carbon slurry. The CNTs employed in this study were prepared by a catalytic chemical vapor deposition. The vapor growth of CNTs was carried out in a horizontal furnace at $850^{\circ} \mathrm{C}$, using $\mathrm{Ni}$ nanoparticles and ethylene as catalyst and carbon precursor, respectively. The other additive, conductive CB (Super P, Taiwan Maxwave Co., Ltd.) was one of commonly used conducting materials for electrode preparation in Li-ion battery. The fabrication process of the carbon black was based on partial oil oxidation of carbochemical and petrochemical raw materials. The CB powder with a mean size of 20-50 nm displayed high conductivity and purity (>99.5\%). The CNT and CB samples were poured into the MCMB slurry and then dispersed in an ultrasonic bath for $0.5 \mathrm{hr}$, respectively. The weight ratio of MCMB to the carbon additive (i.e., CNT and CB) was set at $90: 10$. After filtration, the carbon composites were placed in a vacuum oven, and the composites were then heated at $105^{\circ} \mathrm{C}$ over night to evaporate ethylene glycol, thus giving dry carbon composite powders.

The procedure for the formation of counter electrode can be descried as follows. Each carbon composite was added into a solution of poly vinylidenefluoride $(\mathrm{PVdF})$ in $\mathrm{N}$ methyl pyrrolidinone (NMP), and the mixture was then mixed at ambient temperature to form an electrode slurry. The PVdF was a binder to adhere the interface between particle-particle and particle-substrate. The mixtures were blended by a 3-D mixer using zirconia balls for $3 \mathrm{hr}$ to prepare uniform slurries. All electrodes were prepared by coating the slurry on ITO glass with a spin coater. To obtain the uniform coating, the coating process consists of two steps: (i) the spinning speed of the first-stage coating process was set at $500 \mathrm{rpm}$ for $5 \mathrm{sec}$, and then (ii) the second-stage speed was raised to $3000 \mathrm{rpm}$ and maintained for $10 \mathrm{sec}$. Then, the electrodes were heated to $120^{\circ} \mathrm{C}$ to evaporate the solvent, NMP, in a vacuum oven overnight. The electrode layers, consisted of $90 \mathrm{wt} . \%$ carbon composite and $10 \mathrm{wt} . \%$ PVdF binder, were adjusted to have a thickness of 5-20 $\mu \mathrm{m}$. The two kinds of carbon counter electrodes, CNTs and CB attached to MCMB powders, are designated to $\mathrm{C} 2$ and $\mathrm{C} 3$ electrodes, respectively. For comparison, Pt nanoparticles were well dispersed over pure MCMB counter electrode by using plasma sputtering. The Pt loading to the whole active material was set at $5 \mathrm{wt} \%$. The Pt-coated MCMB counter electrode was designated to $\mathrm{C} 1$ electrode. The schematic diagrams for different counter electrodes are illustrated in
Figure 1. Table 1 lists these carbon electrodes with various compositions. The morphology of electrode materials was observed by using field-emission scanning electron spectroscope (FE-SEM, JEOL JSM-5600). The crystalline structures of the carbon samples were characterized by X-ray diffraction (XRD, Shimadzu Labx XRD-6000) spectroscopy and Raman spectroscopy (Renishaw Micro-Raman spectrometer).

The photoanode used here was composed of $\mathrm{TiO}_{2}$ powder (P25, Degussa), glacial acetic acid, ethylene cellulose, carbowax, and Triton X-100. A screen printer was applied to coat the $\mathrm{TiO}_{2}$ layer on the ITO substrate with sheet resistance of $10 \Omega /$ square. The treated ITO glass was dried at $80^{\circ} \mathrm{C}$ for $8 \mathrm{hr}$ and then sintered at $450^{\circ} \mathrm{C}$ for $1 \mathrm{hr}$, forming the $\mathrm{TiO}_{2}$ photoelectrodes. The electrolyte composition was composed of lithium iodide $(0.5 \mathrm{M})$, iodine $(0.05 \mathrm{M})$, 4-tertbutylpyridine $(0.5 \mathrm{M})$, and 1-methyl-3-propylimidazolium iodide $(0.6 \mathrm{M})$ in 3-methoxypropionitrile. To ensure the dye adsorption, each photoanode was immersed in $0.3 \mathrm{mM}$ N719/ethanol solution (Solaronix) overnight. The sandwichtype DSCs having an active area of $0.49 \mathrm{~cm}^{2}$ were used to compare their cell performance. The I-V curves of DSCs were examined by an Keithley meter under Oriel solar simulator illumination (AM 1.5, $100 \mathrm{~mW} / \mathrm{cm}^{2}$ ). The light intensity of the illumination source was calibrated by using a standard silicon photodiode.

\section{Results and Discussion}

The crystallographic structure of the carbon samples is observed by XRD analysis. Typical XRD patterns of C1, C2, and C3 electrode materials are depicted in Figure 2. The XRD pattern of fresh MCMB was also showed for comparison. The XRD pattern of fresh MCMB shows a diffraction peak (002) centered at $c a .2 \theta=26.1^{\circ}$, originated from the crystalline nature of graphite. Basically, the interlayer distance, $d_{002}$, is close to $0.335 \mathrm{~nm}$ for highly oriented graphite carbon [15]. The $d_{002}$ value of fresh MCMB is approximately $0.337 \mathrm{~nm}$ according to the calculation of Bragg's equation. It can be seen that the addition of $\mathrm{Pt}, \mathrm{CNT}$, and $\mathrm{CB}$ do not significantly alter the XRD intensity of graphite. The C1 pattern displays several representative $\mathrm{Pt}$ peaks after the $\mathrm{Pt}$ sputtering on MCMB spheres. Both XRD patterns of C2 and C3 carbon composites almost show typical peaks regarding the crystalline graphite at fixed positions, implying that the MCMB matrix still maintains a highly $\mathrm{sp}^{2}$ crystalline structure.

To inspect the influence of additive, Raman spectroscopy is an appropriate technique to analyze the graphite degree of carbon materials. Figure 3 shows the Raman spectra of C1, C2, and C3 electrode materials. The Raman spectrum of fresh MCMB is also used to compare with other three spectra. It is known that the Raman band at $1580 \mathrm{~cm}^{-1}$ is assigned to the stacking of the graphite hexagon network plane ( $G$ band), whereas the band at $1350 \mathrm{~cm}^{-1}$ can be attributed amorphous carbon or deformation vibrations of a hexagonal ring ( $D$ band) $[16]$. The $I_{D} / I_{G}$ intensity ratio has an order as follows: C1 $(0.31)>\mathrm{C} 2(0.26)>\mathrm{C} 3(0.14)$. Excepting for C3 sample, original MCMB has a lower $I_{D} / I_{G}$ 


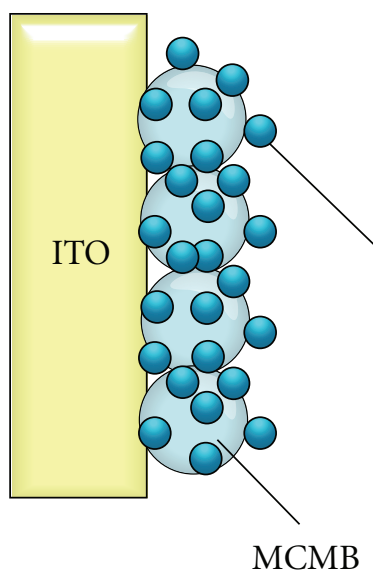

(a)

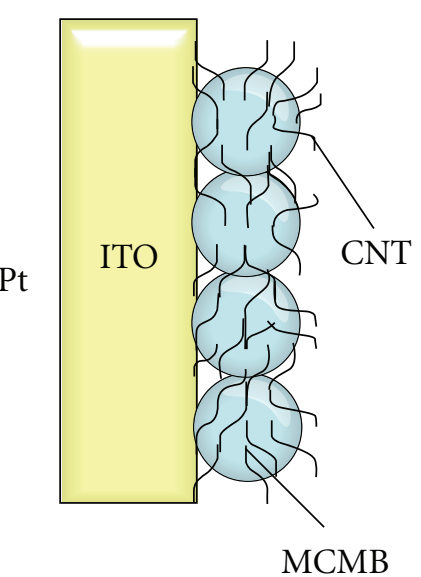

(b)

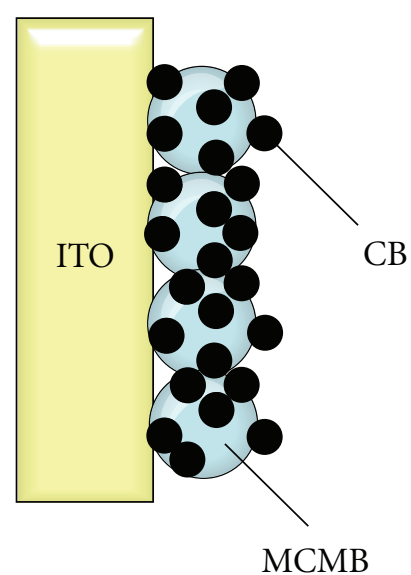

(c)

Figure 1: Schematic diagrams of (a) Pt, (b) CNTs, and (c) CB coated on MCMB powders.

TABLE 1: Parameters for solar cells based on different carbon counter electrodes.

\begin{tabular}{lccccc}
\hline Electrode & Composition $(\mathrm{wt} \%)$ & $I_{\mathrm{SC}}\left(\mathrm{mA} \mathrm{cm}^{-2}\right)$ & $V_{\mathrm{OC}}(\mathrm{V})$ & & $F F$ \\
\hline $\mathrm{C} 1$ & $\mathrm{C}: \operatorname{Pt}(95: 5)$ & 7.45 & 0.69 & 0.62 & 3.17 \\
$\mathrm{C} 2$ & $\mathrm{C}: \mathrm{CNT}(90: 10)$ & 7.68 & 0.74 & 0.52 & 2.96 \\
$\mathrm{C} 3$ & $\mathrm{C}:$ Super P $(90: 10)$ & 7.19 & 0.72 & 0.69 & 3.55 \\
\hline
\end{tabular}

ratio of 0.18 than $\mathrm{C} 1$ and $\mathrm{C} 2$ samples. As to $\mathrm{C} 1$ sample, this increase of $I_{D} / I_{G}$ ratio is presumably due to two reasons: (i) Pt deposits occupy some coverage of crystalline graphite, and (ii) graphite surface may be partially destroyed during the sputtering process. For $\mathrm{C} 2$ sample, this raising $I_{D} / I_{G}$ ratio can be attributed to the fact that few imperfect defects or surface oxides are attached to the sidewall or both ends of CNTs, inducing the disorder degree in the carbon composites.

Figures 4(a), 4(b), and 4(c) shows top-view FE-SEM images of C1, C2, and C3 carbon counter electrodes at the same magnification, respectively. As shown in Figures 4(a) and 4(d), Pt deposits are well dispersed and coated over the surface of graphite. The inset of this figure clearly reflects that as-deposited Pt has an average size of 5-10 $\mathrm{nm}$ and a narrow particle size distribution. As for C2 sample, a large number of CNTs cover the graphite surface, forming a nanomicro junction hybrid. The CNTs possess an average diameter of $50-80 \mathrm{~nm}$ and a length of several micrometers. The onedimensional CNTs are coiled tube shape, indicating that some carbon atoms randomly stack and thus form some imperfections on the sidewall of nanotubes, which has been demonstrated elsewhere $[16,17]$. The FE-SEM image of C3 sample shows a large amount of CB nanoparticles attached to graphite powders. The size of the particulates ranges from 20 to $50 \mathrm{~nm}$. These particulates seem like nanosized tips that cover the graphite matrix.

The IPCE spectra for the DSCs fabricated with various carbon-based counter electrodes are illustrated in Figure 5. The spectra apparently reveal that the DSCs typically display a similar distribution but different photon to current efficiencies within the wavelength region of $300-800 \mathrm{~nm}$. The maximum IPCE takes place at approximately $530 \mathrm{~nm}$ for all DSCs, indicating the nature of the N719 dye. It is found that C2 counter electrode exhibits the highest IPCE response than other two electrodes in the whole region of wavelength. This can be presumably due to the amount of dye adsorption. The porosity of the carbon composites can be determined by physisorption of nitrogen at $-196^{\circ} \mathrm{C}$. The BrunauerEmmett-Teller (BET) surface areas have an order as follows: C2 $\left(50.6 \mathrm{~m}^{2} / \mathrm{g}\right)>\mathrm{C} 3\left(12.6 \mathrm{~m}^{2} / \mathrm{g}\right)>\mathrm{C} 1\left(4.5 \mathrm{~m}^{2} / \mathrm{g}\right) \approx$ original $\operatorname{MCMB}\left(2.1 \mathrm{~m}^{2} / \mathrm{g}\right)$. This finding demonstrates that the CNT additive offers more surface area accessible for the organic adsorption, raising the dye capacity per unit area of electrode. Basically, original graphite powder is a nonporous solid media after high-temperature graphitization. As for the two carbons, an increase of BET surface area may be due to (i) nanosized particles and (ii) nanovoids or nanocavities from the particle aggregation. However, it is worth noting that the IPCE intensity is not proportional to the BET surface area. This discovers two crucial messages: (i) the dye affinities to carbon and to platinum are different, and (ii) the inner surface of CNTs cannot be completely wetted by dye molecule, thus inducing the low surface accessibility.

Figure 6 shows the typical photocurrent-voltage curves of DSCs fabricated with various carbon electrodes. The performance curves reflect different photovoltaic behaviors, originated from the effect of counter electrodes. To evaluate the cell performance, the power conversion efficiency $(\eta)$ of 


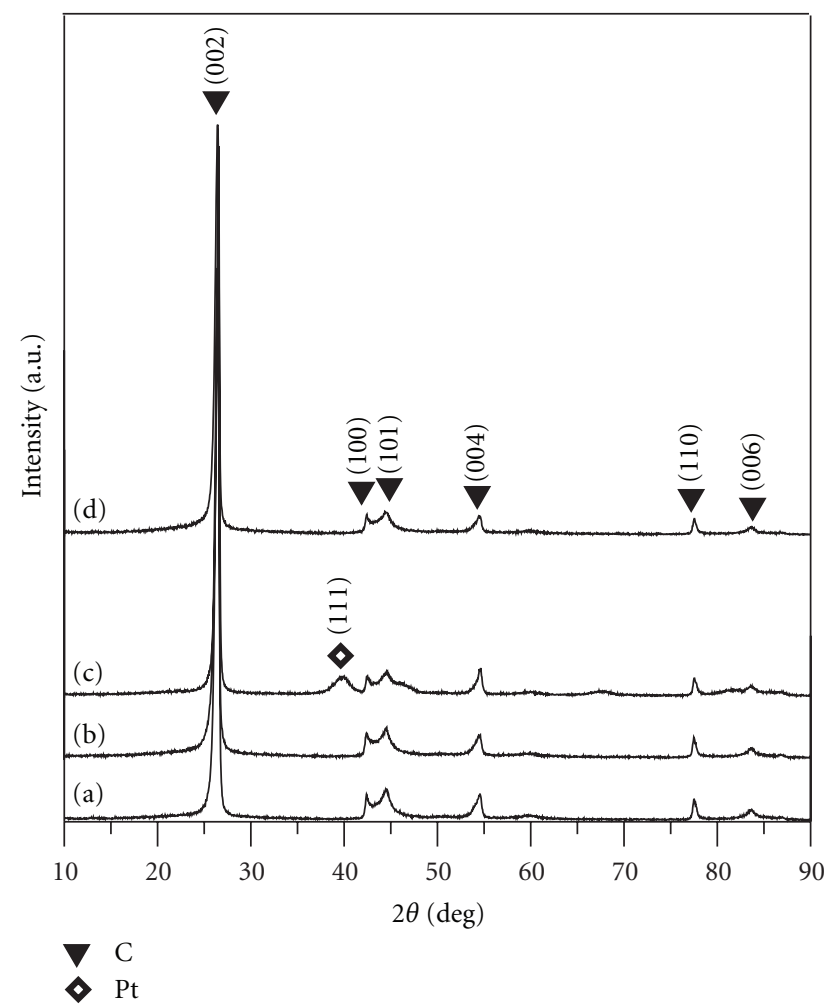

FIgure 2: Typical XRD pattern of different carbon counter electrodes: (a) MCMB, (b) C1, (c) C2, and (d) C3.

the DSC can be calculated by

$$
\eta(\%)=\frac{V_{\mathrm{OC}} I_{\mathrm{SC}} F F}{P_{\text {in }} A} \times 100,
$$

where $I_{\mathrm{SC}}$ is short-circuit current, $V_{\mathrm{OC}}$ is open-circuit voltage, $F F$ is fill factor, $A$ is active area of photoelectrode, and $P_{\text {in }}$ is incident light power $\left(100 \mathrm{~mW} / \mathrm{cm}^{2}\right.$ in this study). The $F F$ value is obtained from

$$
F F=\frac{V_{m} I_{m}}{V_{\mathrm{OC}} I_{\mathrm{SC}}},
$$

where $V_{m}$ and $I_{m}$ are voltage and photocurrent at maximum output power point, respectively. Table 1 summarizes the cell performance with different counter electrodes, according to the calculations from (1) and (2). All DSCs exhibit a fairly high $F F$ value $(>0.69 \mathrm{~V})$ but quietly different other performance factors. This difference among these counter electrodes is significantly related to the redox reaction on the design of counter electrodes.

The mechanism on the counter electrodes consists of three steps: (i) ionic diffusion, (ii) catalytic $I^{-} / I_{3}{ }^{-}$reaction, and (iii) charge transfer at interface between counter electrode and electrolyte. The reduction reaction on the surface of counter electrode can be written as $I_{3}{ }^{-}+2 \mathrm{e}^{-} \rightarrow 3 I^{-}$, whereas the oxidation of iodide occurs on the dye molecule $[7,10]$. Accordingly, the charge-transfer reactions at the interfaces of electrolyte/counter electrode and electrolyte/dye molecule are so crucial to influence the efficiency of counter

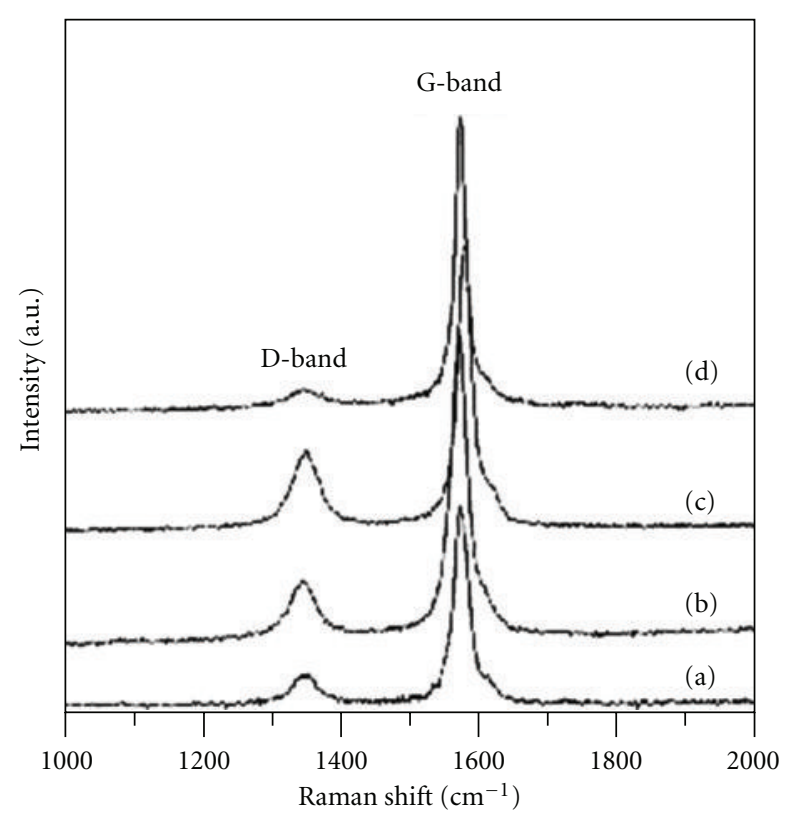

FIGURE 3: Raman spectra of different carbon counter electrodes: (a) MCMB, (b) C1, (c) C2, and (d) C3.

electrodes. Basically, the reduction reaction (i.e., $I^{-} / I_{3}{ }^{-}$) would take place at catalytic sites on the carbon electrodes [11]. These active sites usually locate on some defects of sidewalls or edges of carbon crystals. Thus, the number of defect sites on the carbon electrodes acts an important role in affecting the power performance of DSCs. Since the MCMB powder exhibits highly oriented crystal (see Figures 2 and 3), thus there are a less number of defect sites for the catalytic reaction. However, the microsized graphite offers an excellent medium that allows charge transfer from the ITO substrate to the dye molecule. The active materials such as $\mathrm{Pt}$, CNT, and nanosize $\mathrm{CB}$ act as an active site provider for the redox reaction, as illustrated in Figure 1.

As shown in Table 1, the $\eta$ value for all DSCs displays an order as follows: C3 $(3.55 \%)>\mathrm{C} 1(3.17 \%)>\mathrm{C} 2(2.96 \%)$. This result demonstrates that both CNTs and CB are capable of generating a number of catalytic sites for the redox reaction, similar to $\mathrm{Pt}$ deposits. In comparison, the DSC equipped with $\mathrm{CB}$ counter electrode (C3 electrode) has the greatest $\eta$ and $F F$ values. It is of interest that as shown in Figure 6, C2 electrode represents the highest IPCE response but the lowest power conversion. This can be presumably due to a large amount of dye adsorbed on interior or surface structure of CNTs. However, only low surface coverage of dye adsorbed can be utilized owing to less number of catalytic sits and longer pathway of electron transportation through onedimensional pathway, comparing with zero-dimensional CB nanoparticles. However, these $\mathrm{CB}$ particles not only offer a large number of catalytic dots but also short charge-transfer path, thus inducing fast redox kinetics. On the basis of the cell performance, three designs of counter electrodes show a commercial feasibility to replace expansive Pt film counter 

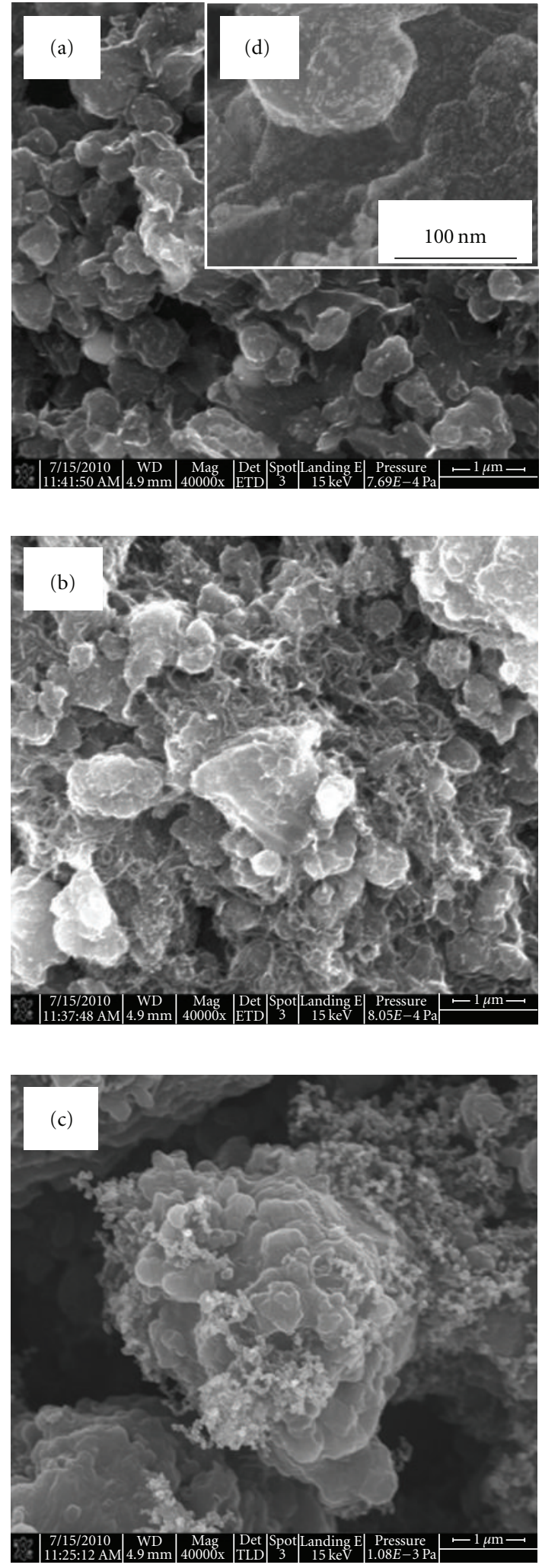

Figure 4: SEM images for (a) Pt, (b) CNTs, and (c) CB coated on MCMB powders. (d) The FE-SEM shows the dispersion of $\mathrm{Pt}$ nanoparticles over MCMB surface.

electrodes in DSC devices. However, to improve the power conversion of DSCs, an optimal factor combination such as

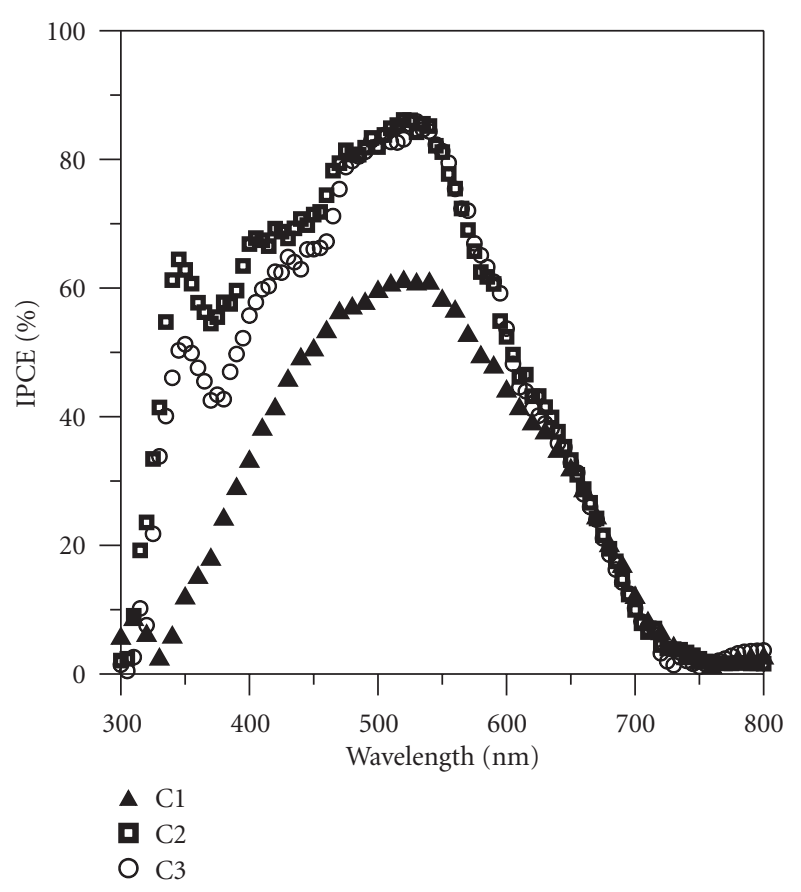

FIgure 5: IPCE spectra for the DSCs fabricated with different counter electrodes.

loading of active carbon materials and dispersion degree still requires a significant investigation.

\section{Conclusions}

We have investigated the performance of DSCs fabricated with different MCMB-based counter electrodes, including $\mathrm{Pt}, \mathrm{CNT}$, and $\mathrm{CB}$ additives. The three types of nanosized materials were well dispersed over the graphite matrix, inducing an increased porosity for the dye adsorption. The MCMB offered an excellent charge transfer medium between active material and ITO substrate, whereas the attached Pt deposits, CNTs, and CB nanopowders provided a number of catalytic sites for the $\mathrm{I}^{-} / \mathrm{I}_{3}{ }^{-}$reduction. The DSC using the CNT counter electrode displayed the improved IPCE response but the lower power conversion efficiency, comparing with the other two DSCs. This can be attributed to two explanations: (i) the dye affinities to carbon and to platinum are different, and (ii) the inner surface of CNTs cannot be completely wetted by dye molecule, thus inducing the low surface accessibility. For comparison, the CB decoration over MCMB not only generated a large number of catalytic sites but also rapid reaction kinetics, thus leading a higher power conversion. On the basis of the above results, such design of carbon counter electrode has been proven to be an attractive candidate for replacing Pt counter electrode in the DSCs.

\section{Acknowledgment}

The authors are very grateful for the financial support from the National Science Council of the Republic of China under 


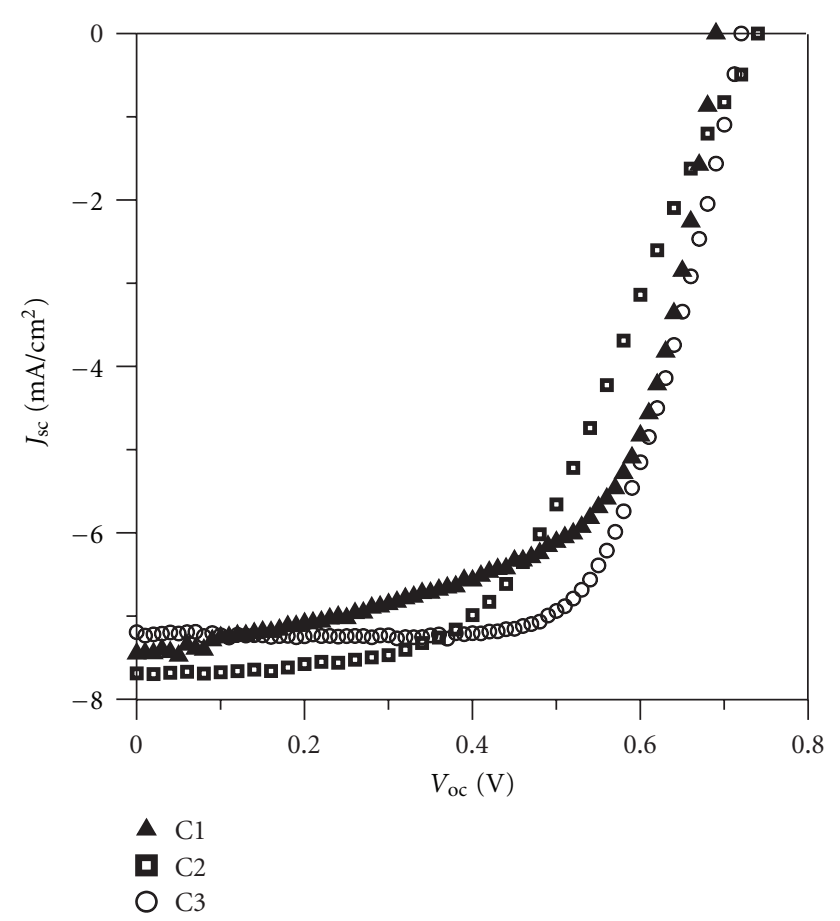

Figure 6: Photocurrent-voltage curves for the DSCs fabricated with different counter electrodes.

the Contracts NSC 100-2120-M-155-001 and NSC 1002221-E-155-031.

\section{References}

[1] H. Choi, H. Kim, S. Hwang, W. Choi, and M. Jeon, "Dyesensitized solar cells using graphene-based carbon nano composite as counter electrode," Solar Energy Materials and Solar Cells, vol. 95, no. 1, pp. 323-325, 2011.

[2] N. Yang, J. Zhai, D. Wang, Y. Chen, and L. Jiang, "Twodimensional graphene bridges enhanced photoinduced charge transport in dye-sensitized solar cells," ACS Nano, vol. 4, no. 2, pp. 887-894, 2010.

[3] W. J. Lee, E. Ramasamy, D. Y. Lee, and J. S. Song, "Performance variation of carbon counter electrode based dye-sensitized solar cell," Solar Energy Materials and Solar Cells, vol. 92, no. 7, pp. 814-818, 2008.

[4] W. J. Lee, E. Ramasamy, D. Y. Lee, and J. S. Song, "Efficient dyesensitized solar cells with catalytic multiwall carbon nanotube counter electrodes," ACS Applied Materials \& Interfaces, vol. 1, no. 6, pp. 1145-1149, 2009.

[5] J. Han, H. Kim, D. Y. Kim, S. M. Jo, and S. Y. Jang, "Watersoluble polyelectrolyte-grafted multiwalled carbon nanotube thin films for efficient counter electrode of dye-sensitized solar cells," ACS Nano, vol. 4, no. 6, pp. 3503-3509, 2010.

[6] J. G. Nam, Y. J. Park, B. S. Kim, and J. S. Lee, "Enhancement of the efficiency of dye-sensitized solar cell by utilizing carbon nanotube counter electrode," Scripta Materialia, vol. 62, no. 3, pp. 148-150, 2010.

[7] W. Hong, Y. Xu, G. Lu, C. Li, and G. Shi, "Transparent graphene/PEDOT-PSS composite films as counter electrodes of dye-sensitized solar cells," Electrochemistry Communications, vol. 10, no. 10, pp. 1555-1558, 2008.
[8] J. Chen, K. Li, Y. Luo et al., "A flexible carbon counter electrode for dye-sensitized solar cells," Carbon, vol. 47, no. 11, pp. 2704-2708, 2009.

[9] H. J. Choi, J. E. Shin, G. W. Lee, N. G. Park, K. Kim, and S. C. Hong, "Effect of surface modification of multi-walled carbon nanotubes on the fabrication and performance of carbon nanotube based counter electrodes for dye-sensitized solar cells," Current Applied Physics, vol. 10, no. 2, pp. S165S167, 2010.

[10] M. Y. Yen, C. Y. Yen, S. H. Liao et al., "A novel carbon-based nanocomposite plate as a counter electrode for dye-sensitized solar cells," Composites Science and Technology, vol. 69, no. 13, pp. 2193-2197, 2009.

[11] P. Li, J. Wu, J. Lin, M. Huang, Y. Huang, and Q. Li, "Highperformance and low platinum loading Pt/Carbon black counter electrode for dye-sensitized solar cells," Solar Energy, vol. 83, no. 6, pp. 845-849, 2009.

[12] H. Han, U. Bach, Y. B. Cheng, R. A. Caruso, and C. MacRae, "A design for monolithic all-solid-state dye-sensitized solar cells with a platinized carbon counterelectrode," Applied Physics Letters, vol. 94, no. 10, Article ID 103102, pp. 1-3, 2009.

[13] W. J. Lee, E. Ramasamy, D. Y. Lee, and J. S. Song, "Grid type dye-sensitized solar cell module with carbon counter electrode," Journal of Photochemistry and Photobiology A, vol. 194, no. 1, pp. 27-30, 2008.

[14] S. Lee, W. Seok Choi, and B. Hong, "A comparative study of dye-sensitized solar cells added carbon nanotubes to electrolyte and counter electrodes," Solar Energy Materials and Solar Cells, vol. 94, no. 4, pp. 680-685, 2010.

[15] Q. M. Gong, Z. Li, Y. Wang, B. Wu, Z. Zhang, and J. Liang, "The effect of high-temperature annealing on the structure and electrical properties of well-aligned carbon nanotubes," Materials Research Bulletin, vol. 42, no. 3, pp. 474-481, 2007.

[16] C. T. Hsieh, Y. T. Lin, W. Y. Chen, and J. L. Wei, "Parameter setting on growth of carbon nanotubes over transition metal/alumina catalysts in a fluidized bed reactor," Powder Technology, vol. 192, no. 1, pp. 16-22, 2009.

[17] C. T. Hsieh, Y. T. Lin, J. Y. Lin, and J. L. Wei, "Synthesis of carbon nanotubes over $\mathrm{Ni}$ - and Co-supported $\mathrm{CaCO}_{3}$ catalysts using catalytic chemical vapor deposition," Materials Chemistry and Physics, vol. 114, no. 2-3, pp. 702-708, 2009. 


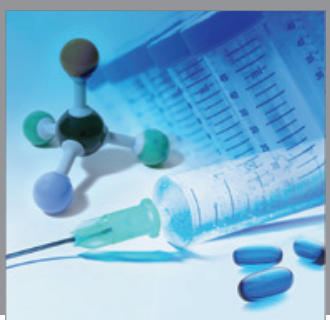

International Journal of

Medicinal Chemistry

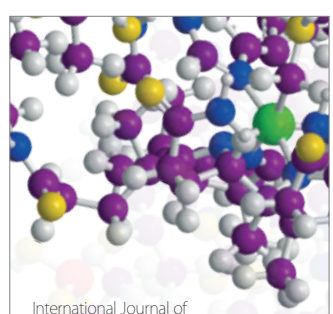

Carbohydrate Chemistry

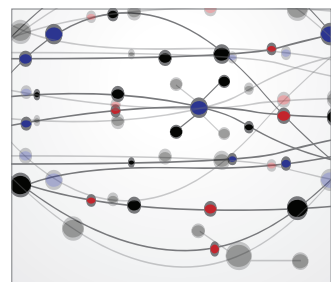

The Scientific World Journal
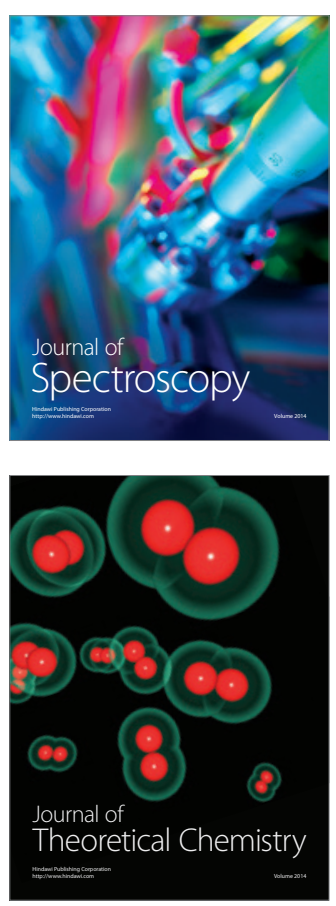
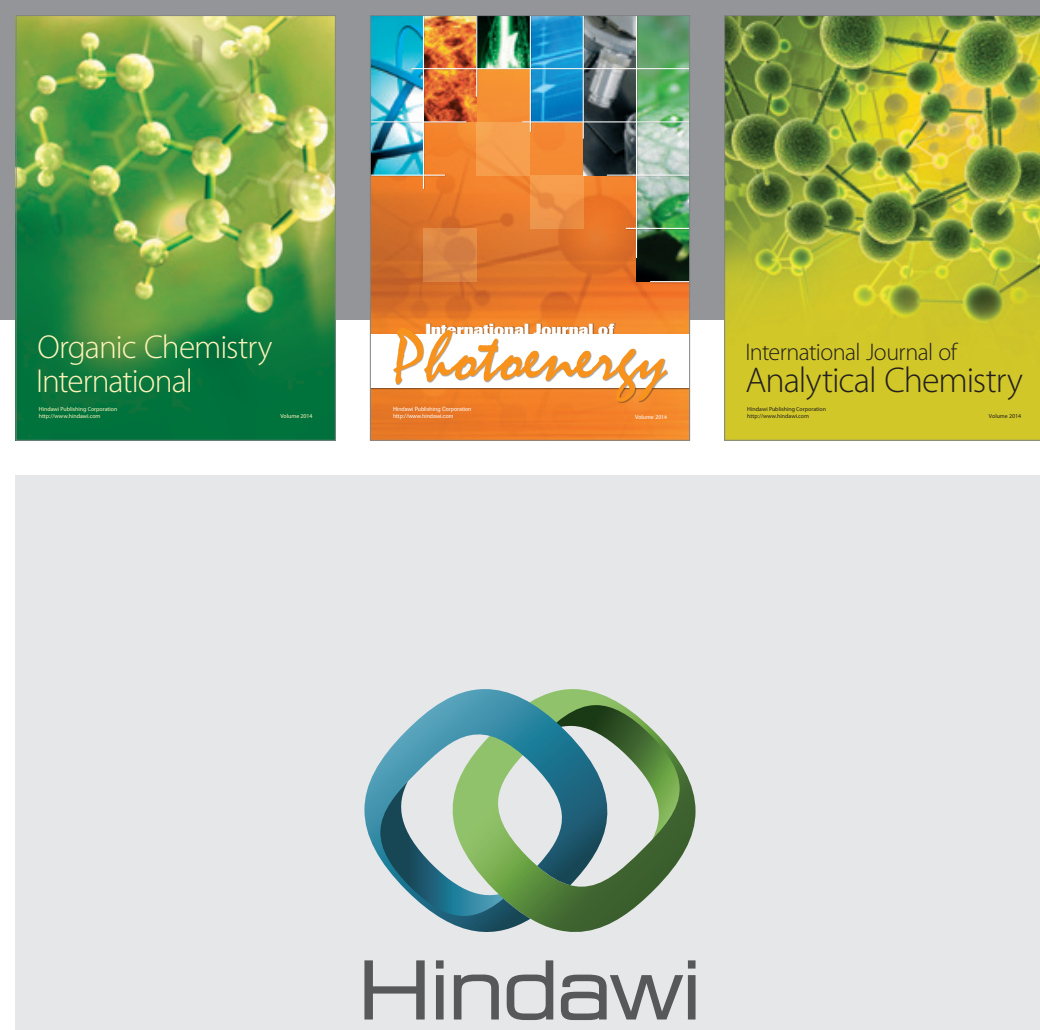

Submit your manuscripts at

http://www.hindawi.com
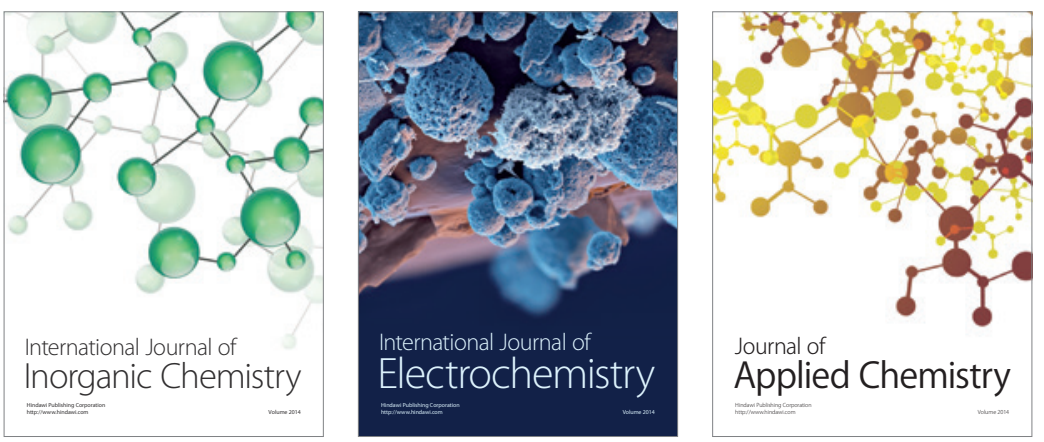

Journal of

Applied Chemistry
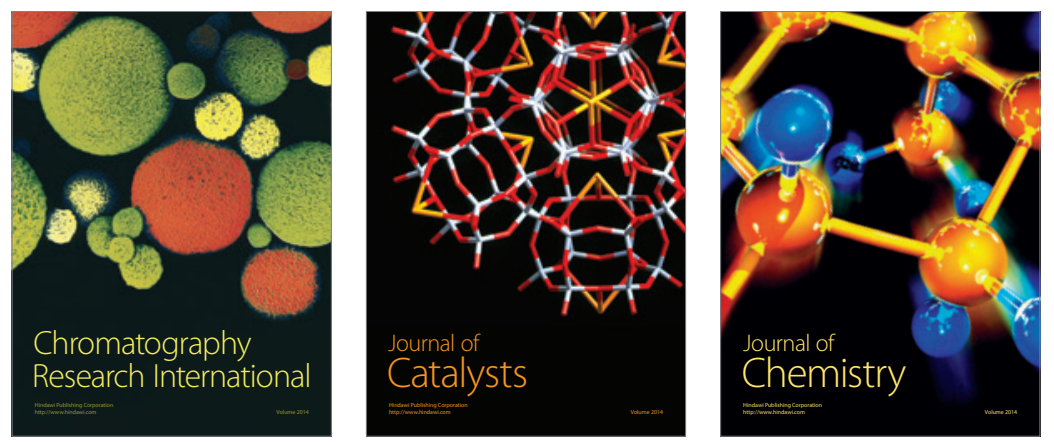
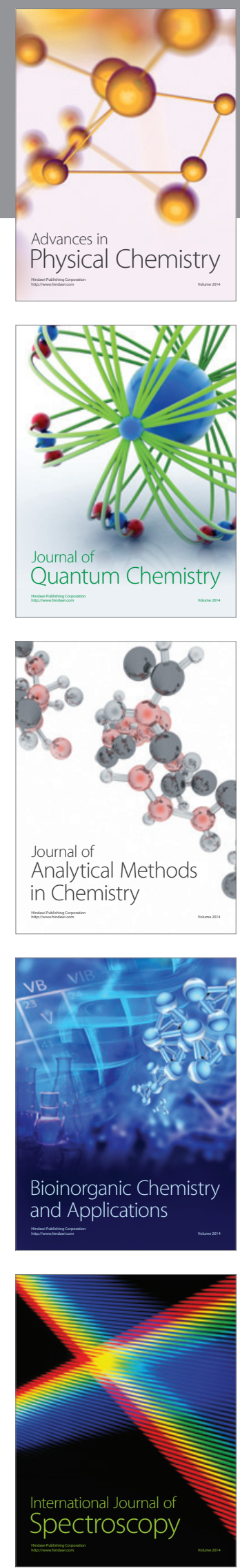